Article

\title{
State of Charge Estimation of Lithium-Ion Batteries Using an Adaptive Cubature Kalman Filter
}

\author{
Bizhong Xia ${ }^{1}$, Haiqing Wang ${ }^{1}$, Yong Tian ${ }^{1,2, *}$, Mingwang Wang ${ }^{3}$, Wei Sun ${ }^{3}$ and Zhihui Xu ${ }^{3}$ \\ 1 Graduate School at Shenzhen, Tsinghua University, Shenzhen 518055, China; \\ E-Mails: xiabz@sz.tsinghua.edu.cn (B.X.); wanghq14@mails.tsinghua.edu.cn (H.W.) \\ 2 College of Optoelectronics Engineering, Shenzhen University, Shenzhen 518060, China \\ 3 Sunwoda Electronic Co. Ltd., Shenzhen 518108, China; E-Mails: lyz@sunwoda.com (M.W.); \\ sunwei@sunwoda.com (W.S.); luojie@sunwoda.com (Z.X.) \\ * Author to whom correspondence should be addressed; E-Mail: ytian@szu.edu.cn; \\ Tel./Fax: +86-755-2603-6982.
}

Academic Editor: Haolin Tang

Received: 20 May 2015 / Accepted: 9 June 2015 / Published: 17 June 2015

\begin{abstract}
Accurate state of charge (SOC) estimation is of great significance for a lithium-ion battery to ensure its safe operation and to prevent it from over-charging or over-discharging. However, it is difficult to get an accurate value of SOC since it is an inner sate of a battery cell, which cannot be directly measured. This paper presents an Adaptive Cubature Kalman filter (ACKF)-based SOC estimation algorithm for lithium-ion batteries in electric vehicles. Firstly, the lithium-ion battery is modeled using the second-order resistor-capacitor (RC) equivalent circuit and parameters of the battery model are determined by the forgetting factor least-squares method. Then, the Adaptive Cubature Kalman filter for battery SOC estimation is introduced and the estimated process is presented. Finally, two typical driving cycles, including the Dynamic Stress Test (DST) and New European Driving Cycle (NEDC) are applied to evaluate the performance of the proposed method by comparing with the traditional extended Kalman filter (EKF) and cubature Kalman filter (CKF) algorithms. Experimental results show that the ACKF algorithm has better performance in terms of SOC estimation accuracy, convergence to different initial SOC errors and robustness against voltage measurement noise as compared with the traditional EKF and CKF algorithms.
\end{abstract}


Keywords: Adaptive Cubature Kalman filter; state of charge; lithium-ion battery; electric vehicle

\section{Introduction}

As energy prices soar and environment pollution increases, electric vehicles (EVs) have become greatly considered in the past few years. Lithium-ion battery is currently considered to be the development trend of traction battery and has been widely used in EVs because of its high power and energy density, high voltage, pollution-free, no memory effect, long cycle life and low self-discharge. A battery management system (BMS) is essential for the lithium-ion battery to maximize its performance, ensure its safety and extend its life. Estimation for battery state of charge (SOC) is one of the most key techniques in the BMS, since SOC indicates the remaining capacity in the battery, which is helpful to dispel the diver's range anxiety, predict the battery's peak power capability and improve the battery's safety by preventing it from possible over-charging or over-discharging. Nevertheless, it is difficult to accurately estimate SOC, because SOC is an inner state of each battery cell that cannot be directly measured and is greatly influenced by many factors, including ambient temperature, discharging current and battery aging. Therefore, the battery SOC has to be estimated with specific estimation techniques according to measured battery parameters, such as voltage, current and temperature.

Different approaches have been proposed to predict the battery SOC with the development of EVs. The existing SOC estimation algorithms can be divided into two categories, namely non-model-based type and model-based type. The former is typically based on Ampere-hour (Ah) or Coulomb counting [1,2], open-circuit voltage (OCV) [3-5], electrochemical impedance spectroscopy (EIS) [6,7], artificial neural networks (ANNs) [8-11] and fuzzy-logic (FL) [12,13]. The Ah counting method acquires the SOC by integrating the current over the time. This method is simple and can be easily implemented on-board, therefore it has been widely used in practice. However, as an open-loop estimation algorithm, Ah counting cannot deal with problems caused by measurement noise and inaccurate initial SOC. The OCV-based method obtains the SOC based on an OCV vs. SOC relationship. Unfortunately, this method is inappropriate for online applications since the battery has to be left in open circuit mode for a long time to reach the steady-state before measuring the OCV. Similarly, the EIS-based method estimates the SOC according to internal impedance of the battery and it is only suitable for offline applications. The ANNs- and FL-based methods predict the SOC according to the nonlinear relationship between the battery SOC and its influencing factors obtained by the trained black-box battery models. They do not require detailed knowledge of the battery systems so they can be applied to any battery type. Besides, these methods have excellent performance if the training data is sufficient to cover the total loading conditions. Nevertheless, it is time-consuming and nearly impossible to collect training data covering all of the battery loading conditions.

In order to improve the accuracy of SOC estimation, battery model-based and closed-loop estimation methods have been further developed. Despite requiring a higher computational cost than the Ah method, these methods have merits in terms of self-correcting, online computing and availability of the dynamic SOC estimation error range. Thus, they are increasingly popular and more 
suitable for real-time application than the other types of SOC estimation methods. A famous and widely used method is the Kalman filter (KF)-based [14-36] algorithm, which was originally developed to estimate state for linear systems. However, the lithium-ion battery is a strong nonlinear and time-variable system. Thus, modified KF algorithms have to be used in order to extend the application of KF in the nonlinear battery systems. Two commonly used types are extended Kalman filter (EKF) [15-28] and unscented Kalman filter (UKF) [29-36]. The EKF transforms a nonlinear system into a linear system by linearizing the nonlinear function on the basis of the first-order Taylor series expansion. However, the linearizing process inevitably causes large linearization error. Besides complicated computation, the Jacobian matrix may lead to the instability of the filter and inaccurate estimation for highly nonlinear battery systems. In addition, the case will be severer when more complicated OCV-SOC relationships are used and it increases the computational burden of the hardware. Unlike the EKF, the UKF introduces an unscented transformation to approximate the state distribution through a set of sample points, called sigma points, which capture the mean and covariance of the state distribution. It has been demonstrated that UKF has better performance than EKF in terms of accuracy and robustness [37,38]. Furthermore, UKF does not need to calculate the Jacobian matrix online. Unfortunately, EKF and UKF both suffer from divergence or the curse of dimensionality or both [39].

In 2009, a new nonlinear filter, called cubature Kalman filter (CKF), for high-dimensional state estimation was proposed by Arasaratnam and Haykin [39]. Based on the radial-spherical cubature rule, the CKF uses a set of $2 n$ points, where $n$ represents the state-vector dimension, to capture the mean and covariance of the states of a nonlinear system with additive Gaussian noise. It is considered to be more efficient and stable than the UKF [39-41]. The CKF has been successfully applied in many fields, such as mobile-station locating [42], moving-target tracking [43] and spacecraft attitude estimation [44].

This paper focuses on the application of the CKF in battery SOC estimation. An adaptive rule for the updating of the process and measurement noise covariance is presented to improve the algorithm performance. Two typical driving cycles, including the Dynamic Stress Test (DST) and New European Driving Cycle (NEDC) are applied to assess the performance of the proposed method by comparing with the standard CKF method. The assessment includes estimation accuracy and robustness against measurement noise.

The remainder of this paper is organized as follows. In Section 2, the experimental setup for data acquiring and processing is described. Section 3 derives the battery's state-space equations based on the second-order RC equivalent circuit model, and determines parameters of the battery model using the least-square method. In Section 4, the principle of standard cubature Kalman filter and the adaptive rule for the updating of the process and measurement covariances are introduced in detail. In addition, the implementation of adaptive CKF-based SOC estimation algorithm is presented. The experimental results are discussed in Section 5, and Section 6 makes conclusions of the paper.

\section{Experimental Setup}

The schematic diagram of the battery test bench is shown in Figure 1. It consists of tested lithium-ion battery cells, a programmable power supply for cell charging (ITECH IT6952A, ITECH Electronics, Nanjing, China), a programmable electric load for cell discharging (ITECH IT8510, 
ITECH Electronics, Nanjing, China), a control board for data acquisition, and a host computer for monitoring and processing experimental data. The IT6952A power supply can charge the battery cell with a maximum current of $25 \mathrm{~A}$ at a maximum voltage of $60 \mathrm{~V}$, while the IT8510 electric load is able to provide the maximum discharge current of $20 \mathrm{~A}$ with the maximum voltage of $120 \mathrm{~V}$. The voltage setup accuracy of the IT6952A power supply is within $0.03 \%+5 \mathrm{mV}$, while the error of current sensor of the IT8510 electric load is within $0.1 \%$. Battery voltage and current are measured with a sampling rate of $10 \mathrm{~Hz}$ and transmitted to the host computer every second through RS485 ports. MATLAB R2010a software (MathWorks, Natick, MA, USA) installed in the host computer is used for data processing, such as battery parameters determination and SOC prediction. It is well known that the performance of lithium-ion battery is highly related with the cathode materials, such as $\mathrm{LiFePO}_{4}$, $\mathrm{LiCoO}_{2}, \mathrm{LiMn}_{2} \mathrm{O}_{4}$ and $\mathrm{LiNi}_{x} \mathrm{Co}_{y} \mathrm{Mn}_{z} \mathrm{O}_{2}$, where $x+y+z=1$. Compared with other materials, layered transition-metal oxide $\mathrm{LiNi}_{x} \mathrm{Co}_{y} \mathrm{Mn}_{z} \mathrm{O}_{2}$ has the merits of high energy density, excellent consistency, mild thermal stability, low cost, and low toxicity [45]. Thus, the Samsung $\mathrm{LiNi}_{x} \mathrm{Co}_{y} \mathrm{Mn}_{z} \mathrm{O}_{2}$ ICR18650-22F (Samsung SDI, Seoul, Korea) battery is used in the test. The ICR18650-22F lithium-ion battery has a nominal voltage of $3.6 \mathrm{~V}$ and a nominal capacity of $2.2 \mathrm{Ah}$.

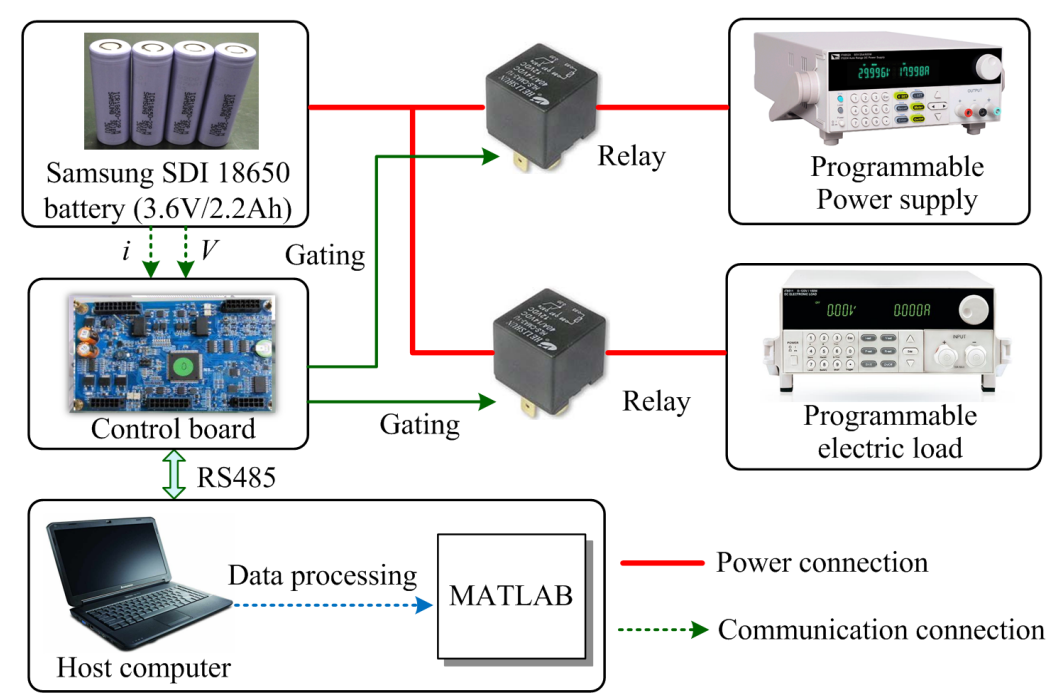

Figure 1. Configuration of the battery test bench.

\section{Battery Modeling and Parameters Identification}

\subsection{Battery Equivalent Circuit Model}

The Kalman filter was developed based on state-space equations of the system and its accuracy is highly dependent on the accuracy of the system model. Thus, a battery model has to be constructed to estimate the SOC using Kalman filter-based approach. There are two basic requirements on a battery model for SOC estimation. Firstly, it can well simulate the dynamic behaviors of the battery. Secondly, the state-space equations can be easily derived according to the model. A commonly used model that well meets the above two requirements is the equivalent circuit model (ECM) with lumped parameters [46]. The most common ECM is comprised of resistor and parallel resistor-capacitor (RC) network(s) connected in series [47]. Although adding more RC networks is a benefit for improving the model accuracy, it leads to the increasing of computation complexity [48]. Accordingly, a tradeoff has 
to be made between the model accuracy and the computational complexity. Herein, the second-order $\mathrm{RC}$ equivalent circuit model is selected to meet the requirement of tradeoff. As shown in Figure 2, the second-order RC battery model consists of an open-circuit voltage $U_{o c}$ (SOC), a resistor $R_{o}$, and two parallel RC networks connected in series. The resistor $R_{o}$ indicates the ohmic resistance caused by the accumulation and dissipation of charge in the electrical double-layer, $R_{p 1}$ and $C_{p 1}$ are the activation polarization resistance and capacitance, respectively, while $R_{p 2}$ and $C_{p 2}$ separately are the concentration polarization resistance and capacitance.

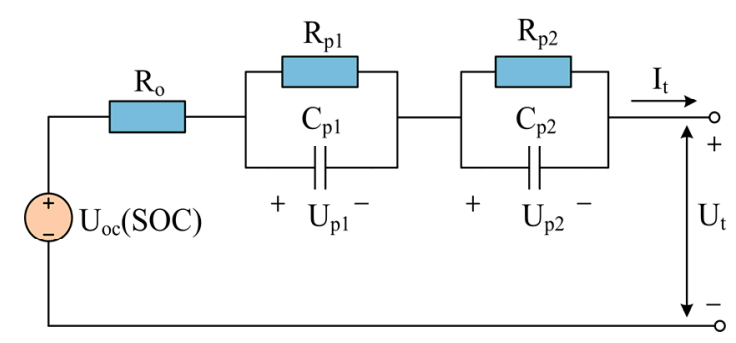

Figure 2. Schematic diagram of the battery equivalent circuit model.

\subsection{State-Space Equations}

The differential equations of the second-order RC equivalent circuit model shown in Figure 2 can be derived as:

$$
\begin{aligned}
& \left\{\begin{array}{l}
S \dot{O} C=-\frac{1}{Q_{n}} I_{t}+w_{1} \\
\dot{U}_{p 1}=-\frac{1}{R_{p 1} C_{p 1}} U_{p 1}+\frac{1}{C_{p 1}} I_{t}+w_{2} \\
\dot{U}_{p 2}=-\frac{1}{R_{p 2} C_{p 2}} U_{p 2}+\frac{1}{C_{p 2}} I_{t}+w_{3}
\end{array}\right. \\
& U_{t}=U_{o c}(S O C)-U_{p 1}-U_{p 2}-R_{o} I_{b}+v
\end{aligned}
$$

where $Q_{n}$ is the battery nominal capacity; $U_{p 1}$ and $U_{p 2}$ are the terminal voltage of $C_{p 1}$ and $C_{p 2}$, respectively; $U_{t}$ and $I_{t}$ are the battery terminal voltage and current, respectively; $U_{o c}$ represents the open circuit voltage $(\mathrm{OCV})$, which is varied with change of SOC value; $\mathrm{w}_{1}, w_{2}$ and $w_{3}$ are the process noise for $S O C, U_{p 1}$ and $U_{p 2}$, respectively; and $v$ represents the measurement noise.

By selecting $x=\left[S O C, U_{p 1}, U_{p 2}\right]^{T}$ as the state vector, and considering the current $I_{t}$ and voltage $U_{t}$ as the input and output variables respectively, the discrete-time state equations of the second-order RC battery model can be obtained as:

$$
\begin{gathered}
x_{k}=f\left(x_{k-1}, u_{k}\right)+w_{k} \\
y_{k}=h\left(x_{k}, u_{k}\right)+v_{k}
\end{gathered}
$$

where $x_{k}$ represents the immeasurable state vector at time step $k ; u_{k}\left(=I_{t, k}\right)$ stands for the input vector; $y_{k}\left(=U_{t, k}\right)$ is the observed output; $v_{k}\left(=\left[\begin{array}{lll}v_{1, k} & v_{2, k} & v_{3, k}\end{array}\right]^{T}\right)$ and $w_{k}$ are separately the process and measurement noises, which are both uncorrelated zero-mean Gaussian white sequences; $f(\cdot)$ and $h(\cdot)$ indicate the 
process and measurement functions, respectively. Generally, $f(\cdot)$ is linear while $h(\cdot)$ is nonlinear due to the nonlinear relationship between the OCV and SOC, which will be illustrated in Section 3.3.

\subsection{Parameters Identification with Forgetting Factor Least-Squares Algorithm}

As shown in Figure 2, parameters needed to be determined include the OCV-SOC equation, $R_{o}, R_{p 1}$, $C_{p 1}, R_{p 2}$ and $C_{p 2}$. In order to acquire the data used to determine the OCV-SOC relationship, a sequence of discharging experiments were carried out. The measured data and fitted curves using the sixth-order polynomial, shown in Equation (5) [49], are shown in Figure 3. It is clear that the fitted equation can well simulate the nonlinear relationship between OCV and SOC.

$$
\begin{aligned}
O C V= & 14.7958 \times S O C^{6}-36.6148 \times S O C^{5}+29.2355 \times S O C^{4}- \\
& 6.2817 \times S O C^{3}-1.6476 \times S O C^{2}+1.2866 \times S O C+3.4049
\end{aligned}
$$

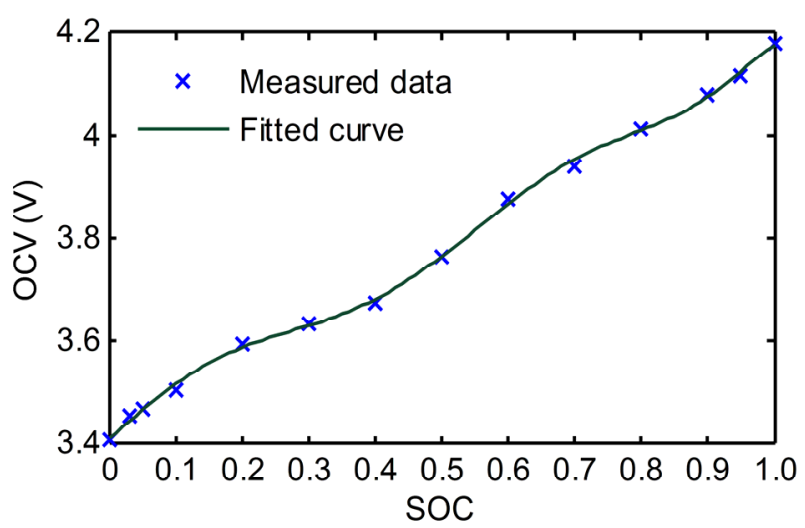

Figure 3. Measured and fitted OCV (open circuit voltage) vs. SOC (state of charge).

Other parameters consisting of $\left\{R_{o}, R_{p 1}, C_{p 1}, R_{p 2}, C_{p 2}\right\}$ can be determined using the least-square method with the following steps:

(i) Calculation of transfer function

By selecting $x=I_{t}$ as the input and $y=U_{o c}-U_{t}$ as the output, the transfer function of the battery model shown in Figure1 can be obtianed as:

$$
G(s)=\frac{Y(s)}{X(s)}=R_{o}+\frac{R_{p 1}}{R_{p 1} C_{p 1} s}+\frac{R_{p 2}}{R_{p 2} C_{p 2} s}
$$

Assuming $\tau_{1}=R_{p 1} C_{p 1}$ and $\tau_{2}=R_{p 2} C_{p 2}$ yields

$$
G(s)=\frac{R_{o} s^{2}+\frac{R_{o}\left(\tau_{1}+\tau_{2}\right)+R_{p 1} \tau_{2}+R_{p 2} \tau_{1}}{\tau_{1} \tau_{2}} s+\frac{R_{o}+R_{p 1}+R_{p 2}}{\tau_{1} \tau_{2}}}{s^{2}+\frac{\tau_{1}+\tau_{2}}{\tau_{1} \tau_{2}} s+\frac{1}{\tau_{1} \tau_{2}}}
$$

(ii) Discretization

Using the bilinear transform rule ( $s=\frac{2}{T} \frac{1-z^{-1}}{1+z^{-1}}$, where $T$ is the sample time) [50], the transfer function in Equation (7) can be discretized as: 


$$
G\left(z^{-1}\right)=\frac{b_{0}+b_{1} z^{-1}+b_{2} z^{-2}}{1+a_{1} z^{-1}+a_{2} z^{-2}}
$$

where $b_{0} b_{1}, b_{2}, a_{1}$ and $a_{2}$ are undetermined coefficients.

Then, the time-domain difference equation of Equation can be expressed as:

$$
y(k)=-a_{1} y(k-1)-a_{2} y(k-2)+b_{0} x(k)+b_{1} x(k-1)+b_{2} x(k-2)
$$

(iii) Resolving

According to the forgetting factor least-squares algorithm, the parameter vector of the battery model can be resolved as follows:

$$
\theta=\left(\Psi^{T} \Psi\right)^{-1} \Psi Y
$$

where $\theta=\left[a_{1}, a_{2}, b_{0}, b_{1}, b_{2}\right] ; Y=\left[\lambda^{n-3} y(3), \lambda^{n-4} y(4), \ldots, y(n)\right]^{T}$ is the output sequence, where $n$ is the number of sample data and $\lambda(0<\lambda<1)$ is the forgetting factor [51]; $\Psi=\left[\Psi_{1}, \Psi_{2}, \ldots, \Psi_{n-2}\right]$ is the regress matrix in which $\Psi_{k}=\lambda^{n-2-k}[-y(k+2),-y(k+1), x(k+2), x(k+1), x(k)]^{T}$.

(iv) Parameters calculation

Using the inverse bilinear transform rule $\left(z^{-1}=\frac{2 / T-S}{2 / T+S}\right)[50]$, the discrete transfer function in Equation (8) can be transformed as:

$$
G(s)=\frac{\frac{b_{0}-b_{1}+b_{2}}{1-a_{1}+a_{2}} s^{2}+\frac{4\left(b_{0}-b_{2}\right)}{T\left(1-a_{1}+a_{2}\right)} s+\frac{4\left(b_{0}+b_{1}+b_{2}\right)}{T^{2}\left(1-a_{1}+a_{2}\right)}}{s^{2}+\frac{4\left(1-a_{2}\right)}{T\left(1-a_{1}+a_{2}\right)} s+\frac{4\left(1+a_{1}+a_{2}\right)}{T^{2}\left(1-a_{1}+a_{2}\right)}}
$$

By comparing Equations (7) and (11), it can be derived that:

$$
\begin{gathered}
R_{o}=\frac{b_{0}-b_{1}+b_{2}}{1-a_{1}+a_{2}} \\
\tau_{1} \tau_{2}=\frac{T^{2}\left(1-a_{1}+a_{2}\right)}{4\left(1+a_{1}+a_{2}\right)} \\
\tau_{1}+\tau_{2}=\frac{T\left(1-a_{2}\right)}{1+a_{1}+a_{2}} \\
R_{o}+R_{p 1}+R_{p 2}=\frac{b_{0}+b_{1}+b_{2}}{1+a_{1}+a_{2}} \\
R_{o}\left(\tau_{1}+\tau_{2}\right)+R_{p 1} \tau_{2}+R_{p 2} \tau_{1}=\frac{T\left(b_{0}-b_{2}\right)}{1+a_{1}+a_{2}}
\end{gathered}
$$

By combining of Equations (12)-(16), $\tau_{1}=R_{p 1} C_{p 1}$ and $\tau_{2}=R_{p 2} C_{p 2}$, parameters $\left\{R_{o}, R_{p 1}, C_{p 1}, R_{p 2}, C_{p 2}\right\}$ of the battery model can be determined. Herein, sample data collected from DST cycles are applied to identify the model parameters. The sample data are shown in Figure 4 and the identified parameters are shown in Table 1. 
Table 1. Identified parameters of the battery model.
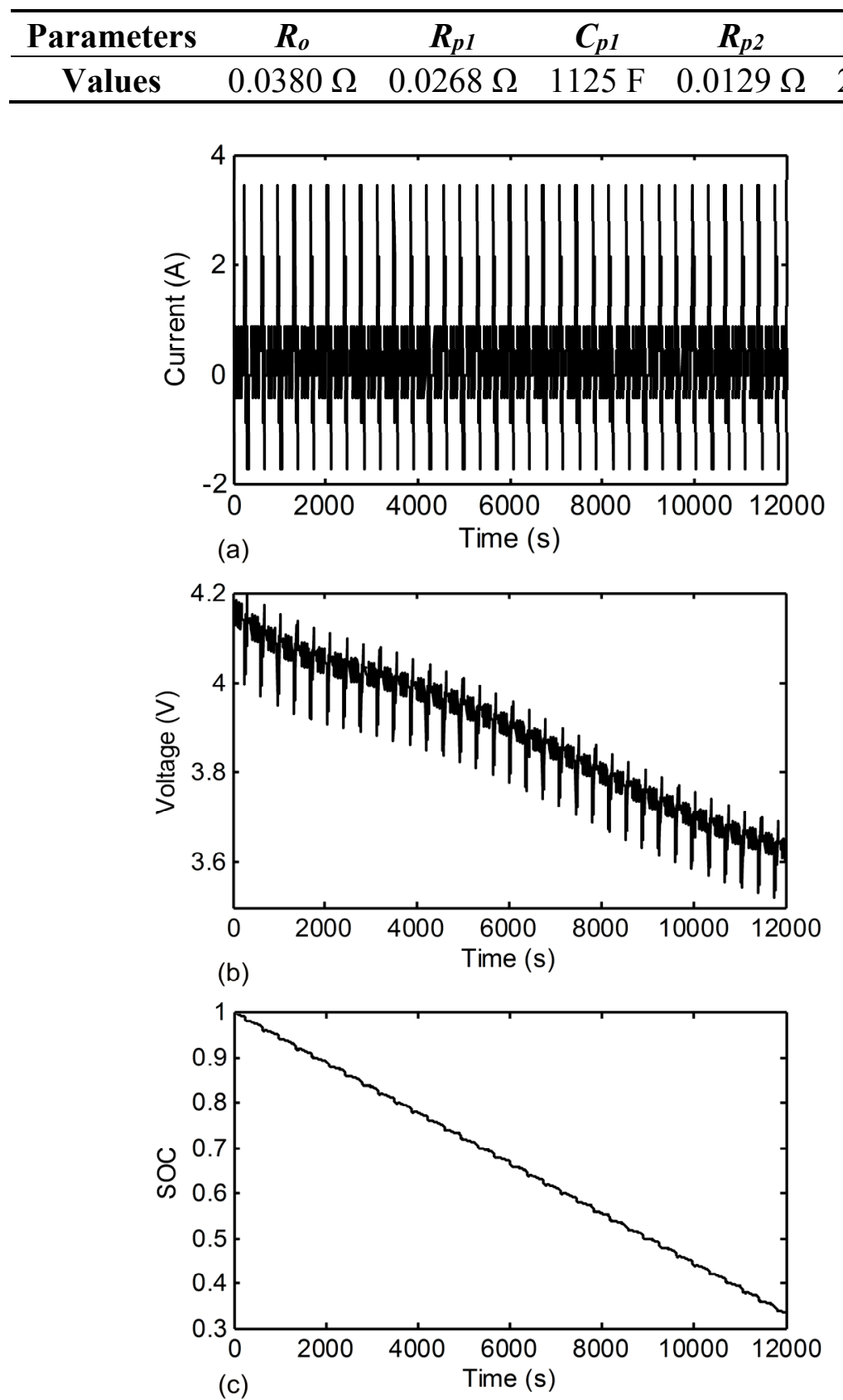

Figure 4. Sample data under DST (Dynamic Stress Test) cycles: (a) current; (b) voltage; (c) SOC.

\subsection{Model Validation}

In order to assess the accuracy of the parameter identification, the measured and estimated battery voltages are compared in Figure 5. A locally enlarged portion of the first three driving cycles is shown in Figure 6a to show more details, and the corresponding voltage error is shown in Figure 6b. It can be seen that the maximum and mean relative errors are about $1.918 \%$ and $0.206 \%$, respectively. Therefore, the battery model can well simulate the dynamic voltage behaviors of the battery. It is worth mentioning that online parameter estimation is helpful to improve the model accuracy since the fact that battery's parameters are related to factors, such as the ambient temperature, operating current and cycling times. However, it is beyond the scope of this paper. 


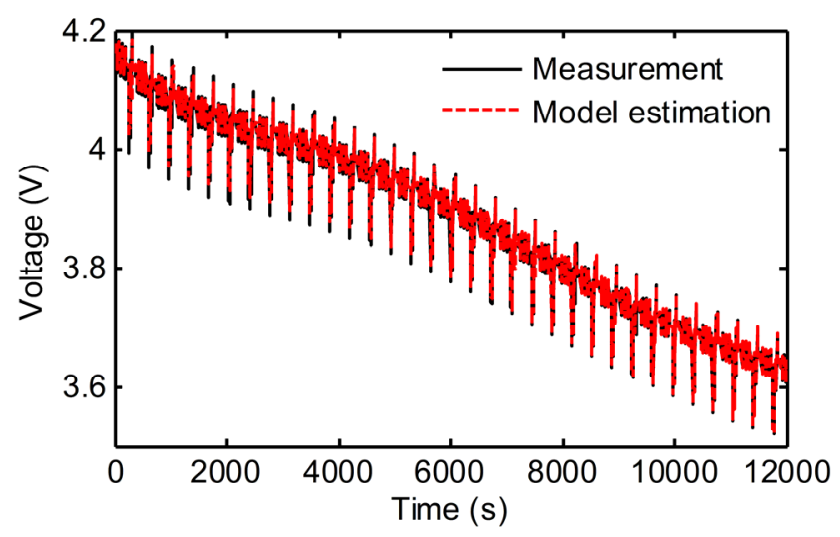

Figure 5. Measured and estimated voltage under DST cycles.

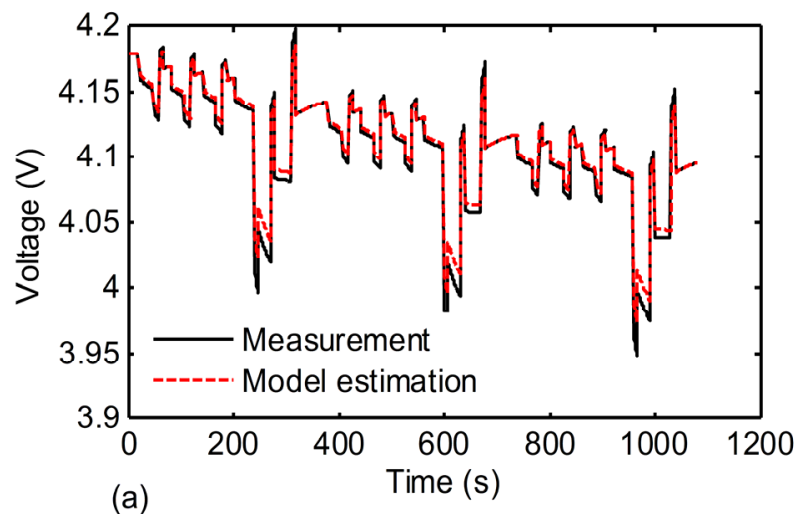

(a)

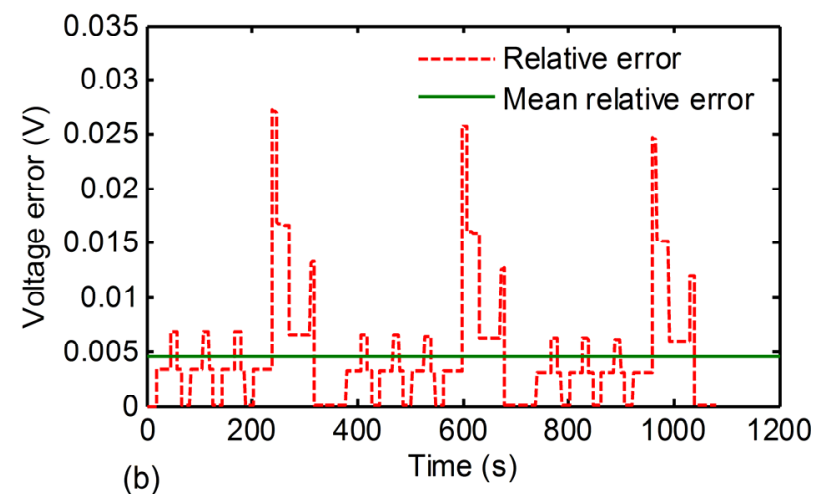

Figure 6. Voltage profiles in the first three DST cycles: (a) voltage; (b) voltage error.

\section{Adaptive Cubature Kalman Filter for SOC Estimation}

The cubature Kalman filter (CKF) was first proposed by Arasaratnam and Haykin in 2009 [39]. It is based on the third-degree spherical-radial cubature rule and uses a set of points to approximate the mean and covariance of the states of a nonlinear system with additive Gaussian noise. The CKF is considered to be more accurate and stable in state estimation than the UKF. A comparison of the efficiency and complexity of the CKF-, EKF-, UKF- and particle filter (PF)-based SOC estimation algorithms for lithium-ion battery has been investigated in [52]. It is concluded that the CKF-based method performs better than both the UKF- and the EKF-based methods. Although the PF-based method has slightly better estimation accuracy compared to the CKF-based method, it is computationally more complex. 
In standard CKF, process noise covariance and measurement noise covariance both are considered to be constant. Nevertheless, it is not the case for a practical battery system in electric vehicles due to random disturbance caused by sensor drift and parameter uncertainties caused by time-varying behaviors of the lithium-ion battery. Therefore, adaptively updating rules for covariance values of the process and measurement noise are required in order to improve performance of the algorithm. In this paper, the idea of covariance matching based on the residual sequence of battery model output voltage proposed in $[31,53]$ is introduced to the $\mathrm{CKF}$, and a residual-based Adaptive Cubature Kalman filter (ACKF) algorithm is developed accordingly. The process of the ACKF algorithm for battery SOC estimation on the basis of battery model is summarized as follows:

(i) Initialization

a. Initial posteriori error covariance: $P_{0}$;

b. Initial process noise covariance: $Q_{0}$;

c. Initial measurement noise covariance: $R_{0}$;

d. Window size for covariance matching: $L_{w}$;

e. Initial mean $\bar{x}_{0}$ and covariance $P_{0}$ with a random state vector $x_{0}$ as follows

$$
\begin{gathered}
\bar{x}_{0}=E\left[x_{0}\right] \\
P_{0}=E\left[\left(x_{0}-\bar{x}_{0}\right)\left(x_{0}-\bar{x}_{0}\right)^{T}\right]
\end{gathered}
$$

(ii) Time update

a. Factorize the error covariance

$$
S_{k-1}=\operatorname{chol}\left(P_{k-1}\right)
$$

where $\operatorname{chol}(\cdot)$ represents a Cholesky decomposition of a matrix returning a lower triangular Cholesky factor. That's to say:

$$
P_{k-1}=S_{k-1} S_{k-1}^{T}
$$

b. Calculate the cubature points

$$
x_{k-1}^{(i)}=S_{k-1} \xi^{(i)}+\hat{x}_{k-1} i=1,2, \cdots, 2 n
$$

where $n$ is the number of state variables and $\xi$ is the set of standard cubature points, which is given by

$$
\xi^{(i)}= \begin{cases}\sqrt{n}[1]^{(i)} & i=1,2, \cdots n \\ -\sqrt{n}[1]^{(i)} & i=n+1, n+2, \cdots 2 n\end{cases}
$$

where [1] represents the identity matrix and $[1]^{(i)}$ denotes its $i$-th column vector. For example, assuming $n=3$ yields:

$$
[1]=\left[\begin{array}{lll}
1 & 0 & 0 \\
0 & 1 & 0 \\
0 & 0 & 1
\end{array}\right]
$$

c. Propagate the cubature points and calculate the predicted state

$$
\chi_{k \mid k-1}^{(i)}=f\left(x_{k-1}^{(i)}, u_{k-1}\right)
$$




$$
\bar{x}_{k \mid k-1}=\frac{1}{2 n} \sum_{i=1}^{2 n} \chi_{k \mid k-1}^{(i)}
$$

d. Calculate the propagated covariance

$$
P_{k \mid k-1}=\frac{1}{2 n} \sum_{i=1}^{2 n}\left(\chi_{k \mid k-1}^{(i)}-\bar{x}_{k \mid k-1}\right)\left(\chi_{k \mid k-1}^{(i)}-\bar{x}_{k \mid k-1}\right)^{T}+Q_{k-1}
$$

where $Q_{k-1}$ is the process noise covariance matrix at time step $k-1$.

(iii) Measurement update

a. Factorize the error covariance

$$
S_{k \mid k-1}=\operatorname{chol}\left(P_{k \mid k-1}\right)
$$

b. Recalculate the cubature points

$$
x_{k \mid k-1}^{(i)}=S_{k \mid k-1} \xi^{(i)}+\hat{x}_{k \mid k-1} i=1,2, \cdots, 2 n
$$

c. Propagate the cubature points and calculate the predicted measurement

$$
\begin{aligned}
& y_{k \mid k-1}^{(i)}=h\left(x_{k \mid k-1}^{(i)}, u_{k}\right) \\
& \bar{y}_{k \mid k-1}=\frac{1}{2 n} \sum_{i=1}^{2 n} y_{k \mid k-1}^{(i)}
\end{aligned}
$$

d. Calculate the estimated covariance

$$
\begin{gathered}
P_{k \mid k-1}^{y}=\frac{1}{2 n} \sum_{i=1}^{2 n}\left(y_{k \mid k-1}^{(i)}-\bar{y}_{k \mid k-1}\right)\left(y_{k \mid k-1}^{(i)}-\bar{y}_{k \mid k-1}\right)^{T}+R_{k-1} \\
P_{k \mid k-1}^{x y}=\frac{1}{2 n} \sum_{i=1}^{2 n}\left(x_{k \mid k-1}^{i}-\bar{x}_{k \mid k-1}\right)\left(y_{k \mid k-1}^{i}-\bar{y}_{k \mid k-1}\right)^{T}
\end{gathered}
$$

where $R_{k-1}$ is the measurement noise covariance matrix at time step $k-1$.

e. Calculate the Kalman gain

$$
K_{k}=P_{k \mid k-1}^{x y}\left(P_{k \mid k-1}^{y}\right)^{-1}
$$

f. Update the predicted state

$$
\hat{x}_{k}=\bar{x}_{k \mid k-1}+K_{k}\left(y_{k}-\bar{y}_{k \mid k-1}\right)
$$

where $y_{k}$ is the measured output at time step $k$.

g. Update the error covariance

$$
P_{k}=P_{k \mid k-1}-K_{k} P_{k \mid k-1}^{y} K_{k}^{T}
$$

(iv) Adjustment of $Q_{k}$ and $R_{k}$

In this step, the process noise covariance $Q$ and measurement noise covariance $R$ are adaptively estimated according to the output voltage residual sequence of the battery model. Thus, $Q$ and $R$ can be iteratively updated as:

$$
Q_{k}=K_{k} F_{k} K_{k}^{T}
$$




$$
R_{k}=F_{k}+\sum_{i=0}^{2 n} W_{n}^{(i)}\left(y_{k \mid k-1}^{(i)}-y_{k}\right)\left(y_{k \mid k-1}^{(i)}-y_{k}\right)^{T}
$$

where $F_{k}$ is an approximation to the covariance of the voltage residual at time step $k$ and is defined as:

$$
F_{k}=\sum_{i=k-L_{w}+1}^{k} e_{i} e_{i}^{T}
$$

where $e_{i}$ is the voltage residual of the battery model at time step $i$, and $L_{w}$ is window size for covariance matching.

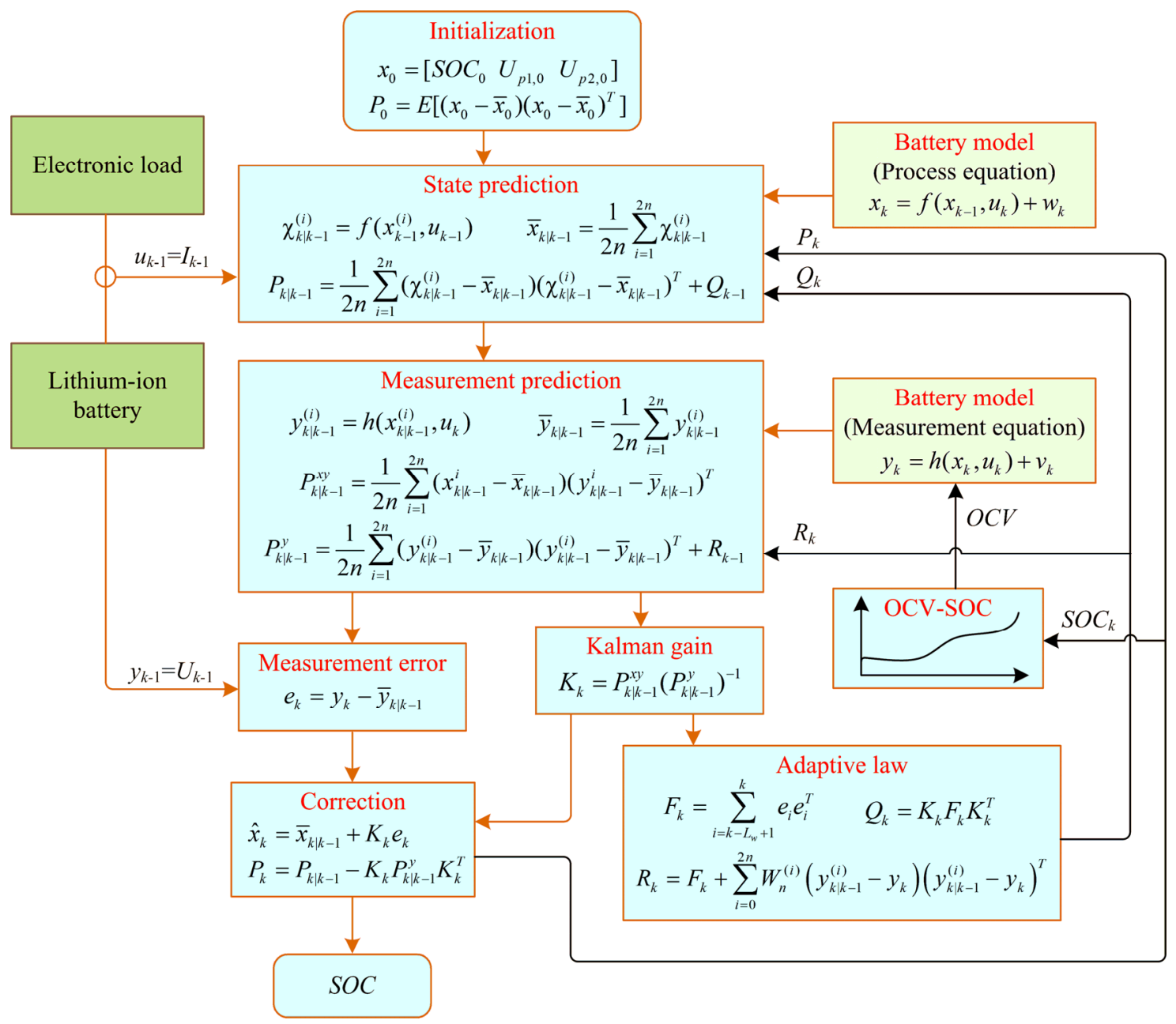

Figure 7. Schematic of the ACKF (adaptive cubature Kalman filter)-based SOC estimation algorithm.

The schematic diagram of the proposed ACKF-based SOC estimation algorithm is shown in Figure 7. After initialization, the estimated state vector $\hat{x}_{k}$ and the estimation error covariance $P_{k}$ can be firstly achieved according to the prediction and update processes. The voltage residual error $e_{k}$ is computed on the basis of the measurement equation of the battery model. Afterwards, the process noise covariance $Q_{k}$ and the measurement noise covariance $R_{k}$ are obtained though the voltage residual-based updating law. Then, $\hat{x}_{k}, P_{k}, Q_{k}$ and $R_{k}$ are used for the next prediction and update processes. The battery SOC can be recursively estimated by repeating the above procedure. 


\section{Results and Discussion}

\subsection{Estimation Results without Measurement Noise}

In this section, experimental data collected from two typical driving cycles, including the $360 \mathrm{~s}$ Dynamic Stress Test (DST) cycle and the 1184 s New European Driving Cycle (NEDC) are applied to evaluate the performance of SOC estimation algorithms under the typical loading conditions of EVs. The profiles under the DST cycles are shown in Figure 4 in Section 3.3, and that under the NEDC cycles are illustrated in Figure 8.
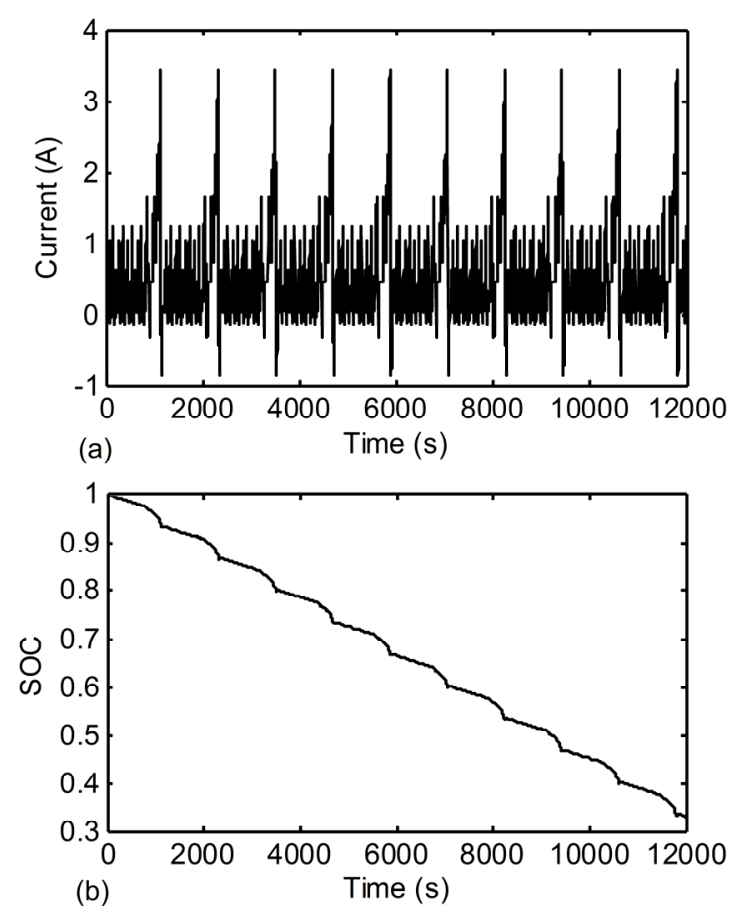

Figure 8. Current and SOC under NEDC (New European Driving Cycle) test: (a) current; (b) SOC.

The proposed method was compared with the widely used EKF and the standard CKF algorithms in terms of estimation accuracy, convergence rate and computational complexity. The comparison results, including root mean square error (RMSE), convergence rate to 5\% SOC error from different initial SOCs and execution time are summarized in Table 2. As an example, the SOC estimation results at $80 \%$ initial SOC under DST and NEDC cycles are separately shown in Figures 9 and 10, where the black solid-line presents the reference SOC computed using Coulomb counting method with accurate current values, the green chain-line represents the estimated value using the EKF algorithm, the blue dotted-line indicates the estimated value using the traditional CKF algorithm, while the red dashed-line describes the estimated value using the proposed adaptive CKF (ACKF) algorithm. It can be found that although the ACKF takes more computational cost, it can improve both the estimation accuracy and convergence rate in comparison with the EKF and CKF algorithms. For example, the RMSE is reduced from $1.2 \%$ to $0.6 \%$ under DST cycles with accurate initial SOC compared with CKF, and from $1.2 \%$ to $0.5 \%$ under NEDC cycles. The average convergence rate can be improved about $20 \%$ compared with EKF, and about $45 \%$ compared with CKF. In addition, the proposed ACKF can reduce the fluctuation of SOC estimation compared with both the EKF and CKF methods. 

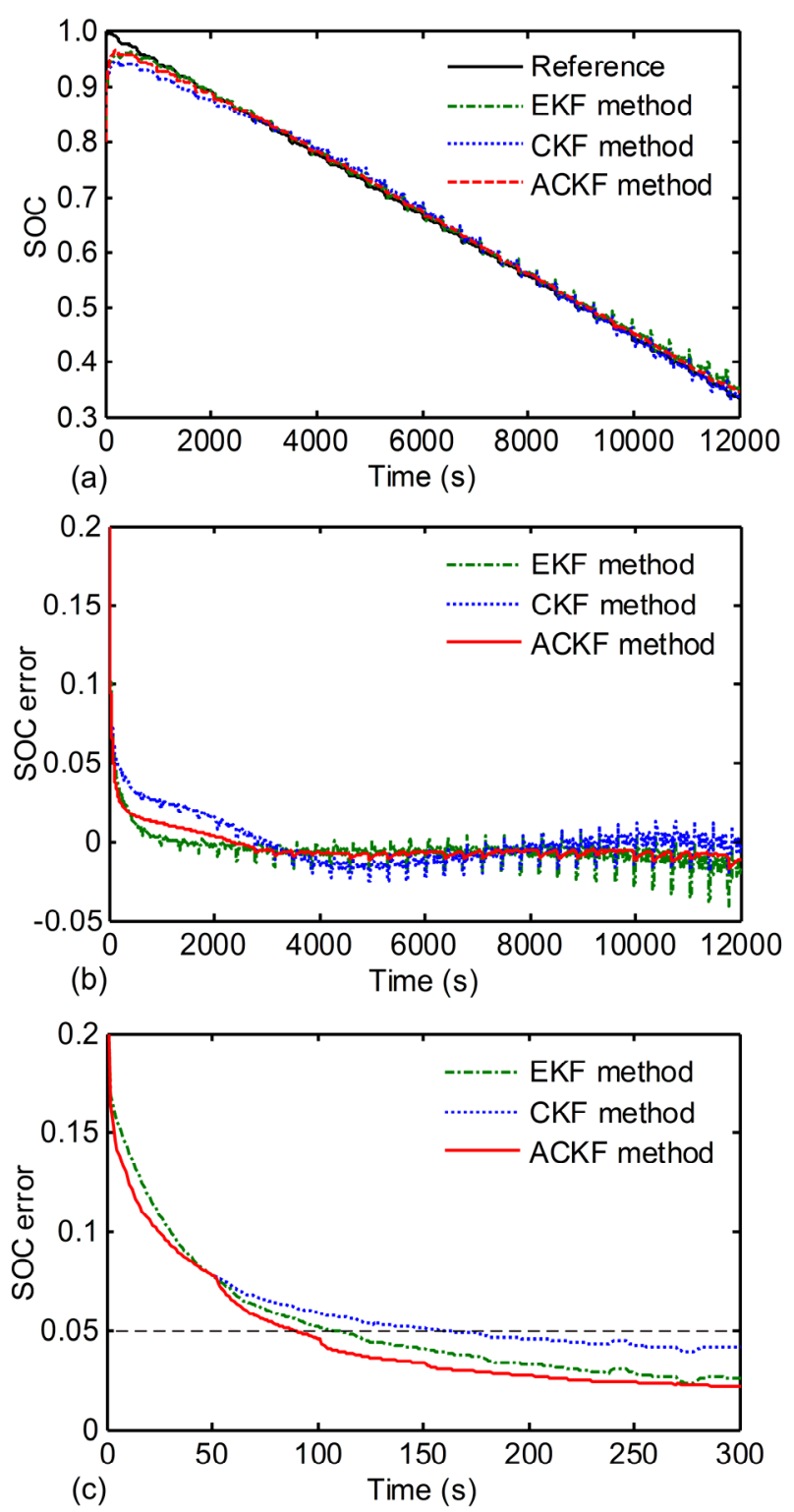

Figure 9. SOC estimation results under DST test: (a) SOC; (b) SOC error; (c) zoom figure for (b).

Table 2. Comparison of SOC estimation without measurement noise.

\begin{tabular}{|c|c|c|c|c|c|c|c|c|}
\hline \multirow[b]{2}{*}{ Methods } & \multirow[b]{2}{*}{$\begin{array}{l}\text { Initial } \\
\text { SOC }\end{array}$} & \multirow[b]{2}{*}{$\begin{array}{l}\text { Execution } \\
\text { time }\end{array}$} & \multicolumn{3}{|c|}{ DST } & \multicolumn{3}{|c|}{ NEDC } \\
\hline & & & $\begin{array}{c}\text { Maximum } \\
\text { error }\end{array}$ & RMSE & $\begin{array}{c}\text { Convergence } \\
\text { rate } \\
\end{array}$ & $\begin{array}{c}\text { Maximum } \\
\text { error }\end{array}$ & RMSE & $\begin{array}{c}\text { Convergence } \\
\text { rate } \\
\end{array}$ \\
\hline \multirow[t]{4}{*}{$\mathrm{EKF}$} & $100 \%$ & $0.76 \mathrm{~s}$ & $4.0 \%$ & $0.8 \%$ & 1 step & $4.3 \%$ & $0.7 \%$ & 1 step \\
\hline & $80 \%$ & & & $1.3 \%$ & 108 step & & $1.2 \%$ & 105 step \\
\hline & $70 \%$ & & & $1.8 \%$ & 205 step & & $1.7 \%$ & 203 step \\
\hline & $60 \%$ & & & $2.3 \%$ & 310 step & & $2.2 \%$ & 270 step \\
\hline \multirow[t]{4}{*}{ CKF } & $100 \%$ & $1.36 \mathrm{~s}$ & $3.8 \%$ & $1.2 \%$ & 1 step & $3.8 \%$ & $1.2 \%$ & 1 step \\
\hline & $80 \%$ & & & $1.6 \%$ & 160 step & & $1.6 \%$ & 155 step \\
\hline & $70 \%$ & & & $2.0 \%$ & 350 step & & $2.0 \%$ & 300 step \\
\hline & $60 \%$ & & & $2.4 \%$ & 405 step & & $2.4 \%$ & 390 step \\
\hline
\end{tabular}


Table 2. Cont.

\begin{tabular}{|c|c|c|c|c|c|c|c|c|}
\hline \multirow[b]{2}{*}{ Methods } & \multirow{2}{*}{$\begin{array}{l}\text { Initial } \\
\text { SOC }\end{array}$} & \multirow{2}{*}{$\begin{array}{l}\text { Execution } \\
\text { time }\end{array}$} & \multicolumn{3}{|c|}{ DST } & \multicolumn{3}{|c|}{ NEDC } \\
\hline & & & $\begin{array}{c}\text { Maximum } \\
\text { error }\end{array}$ & RMSE & $\begin{array}{c}\text { Convergence } \\
\text { rate }\end{array}$ & $\begin{array}{c}\text { Maximum } \\
\text { error }\end{array}$ & RMSE & $\begin{array}{c}\text { Convergence } \\
\text { rate }\end{array}$ \\
\hline \multirow[t]{4}{*}{$\mathrm{ACKF}$} & $100 \%$ & $1.89 \mathrm{~s}$ & $3.8 \%$ & $0.6 \%$ & 1 step & $3.8 \%$ & $0.5 \%$ & 1 step \\
\hline & $80 \%$ & & & $1.2 \%$ & 88 step & & $1.1 \%$ & 90 step \\
\hline & $70 \%$ & & & $1.6 \%$ & 160 step & & $1.5 \%$ & 155 step \\
\hline & $60 \%$ & & & $2.1 \%$ & 255 step & & $2.0 \%$ & 250 step \\
\hline
\end{tabular}
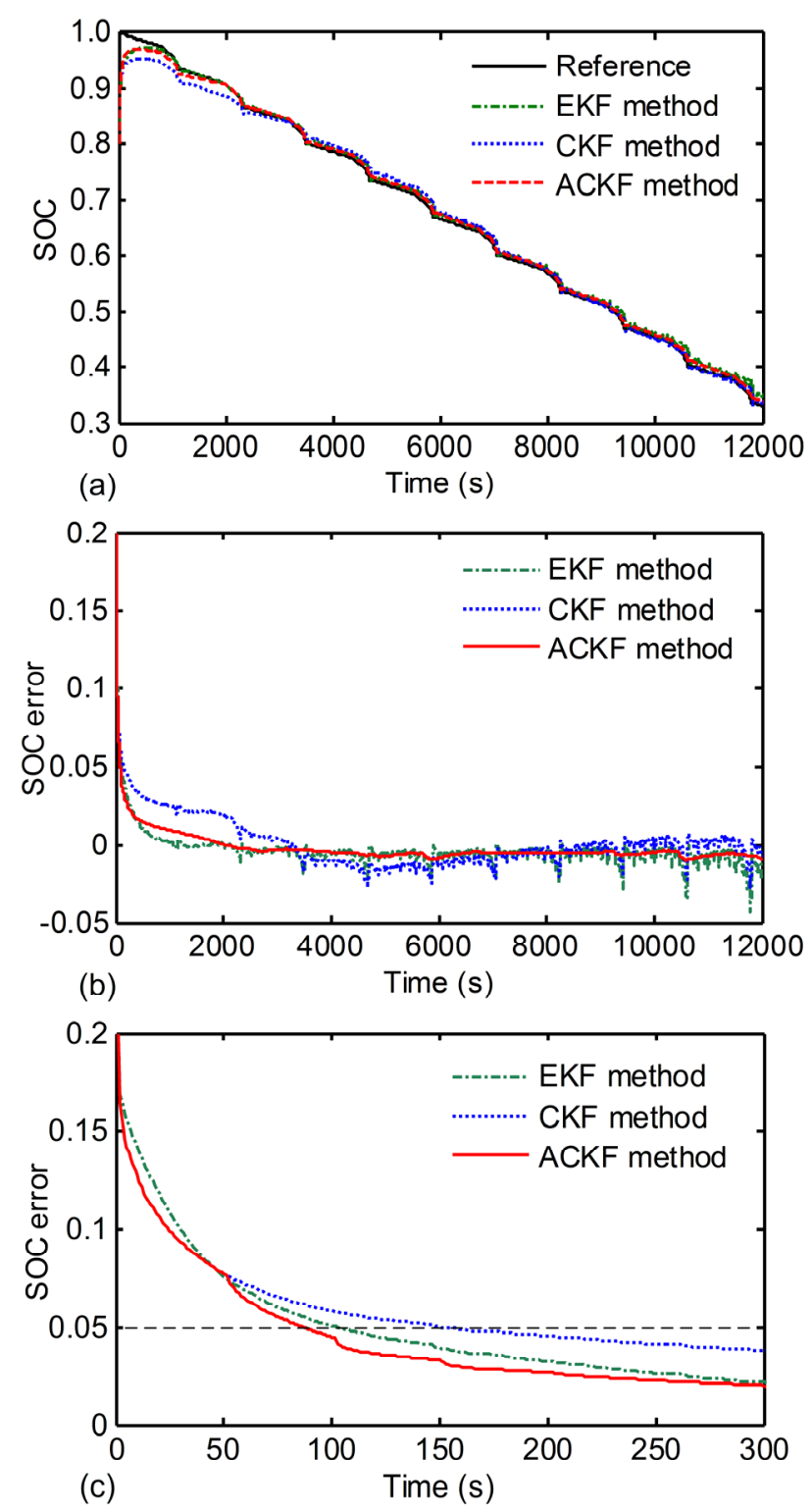

Figure 10. SOC estimation results under NEDC test: (a) SOC; (b) SOC error; (c) zoom figure for (b).

\subsection{Estimation Results with Measurement Noise}

In practice, it is difficult to always get accurate measurement values from an online battery system due to noise caused by factors, such as the electromagnetic interference (EMI) generated by electronic equipment on EVs and temperature drift of sensors. To further evaluate the robustness of the proposed 
ACKF algorithm against measurement noise, a sequence of voltage error shown in Figure 11 is added to the measured voltage. The total voltage error profile $v s$. time is shown in Figure 11a, and a locally enlarged portion is shown Figure $11 \mathrm{~b}$ to illustrate more details. The SOC estimation results with voltage measurement noise under DST and NEDC cycles are shown in Figures 12 and 13, respectively. In both Figures 12 and 13, the black solid-line presents the reference SOC values, the green chain-line represents the estimated SOC using the EKF algorithm, the blue dotted-line indicates the estimated SOC using the traditional CKF algorithm, and the red dashed-line describes the estimated SOC using the ACKF algorithm. The corresponding maximum error and RMSE of SOC estimation are summarized in Table 3.

With all the results shown in Table 3 and Figures 11-13, it is clear that SOC estimation errors using the EKF approach are highly increased due to the introduction of the voltage noise, while those using $\mathrm{CKF}$ and ACKF are slightly increased. For example, the maximum error using EKF increases from $4.0 \%$ to $8.7 \%$, and the RMSE increases from $0.8 \%$ to $3.6 \%$ under DST cycles. However, it is not the case for the $\mathrm{CKF}$ and $\mathrm{ACKF}$ algorithms. As for the ACKF, the maximum error is slightly increased, while the RMSE is even slightly reduced due to the adaptively updating of the voltage covariance. It is accordingly demonstrated that the proposed ACKF algorithm is greatly robust to measurement noise compared with the EKF and CKF algorithms.
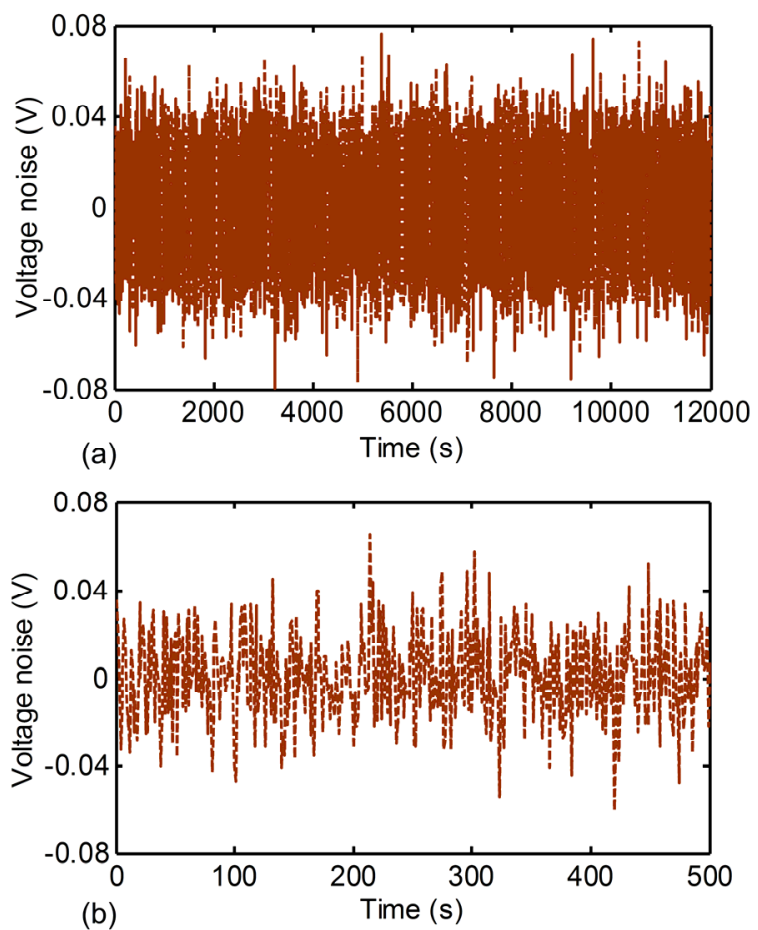

Figure 11. Voltage noise: (a) voltage noise vs. time; (b) zoom figure for (a).

Table 3. Comparison of SOC estimation with measurement noise.

\begin{tabular}{cccccc}
\hline \multirow{2}{*}{ Methods } & \multicolumn{2}{c}{ DST } & & \multicolumn{2}{c}{ NEDC } \\
\cline { 2 - 3 } \cline { 5 - 6 } & Maximum error & RMSE & & Maximum error & RMSE \\
\hline EKF & $8.7 \%$ & $3.6 \%$ & & $9.1 \%$ & $3.7 \%$ \\
CKF & $4.8 \%$ & $1.5 \%$ & & $4.8 \%$ & $1.5 \%$ \\
$\mathrm{ACKF}$ & $4.3 \%$ & $0.5 \%$ & & $4.3 \%$ & $0.4 \%$ \\
\hline
\end{tabular}



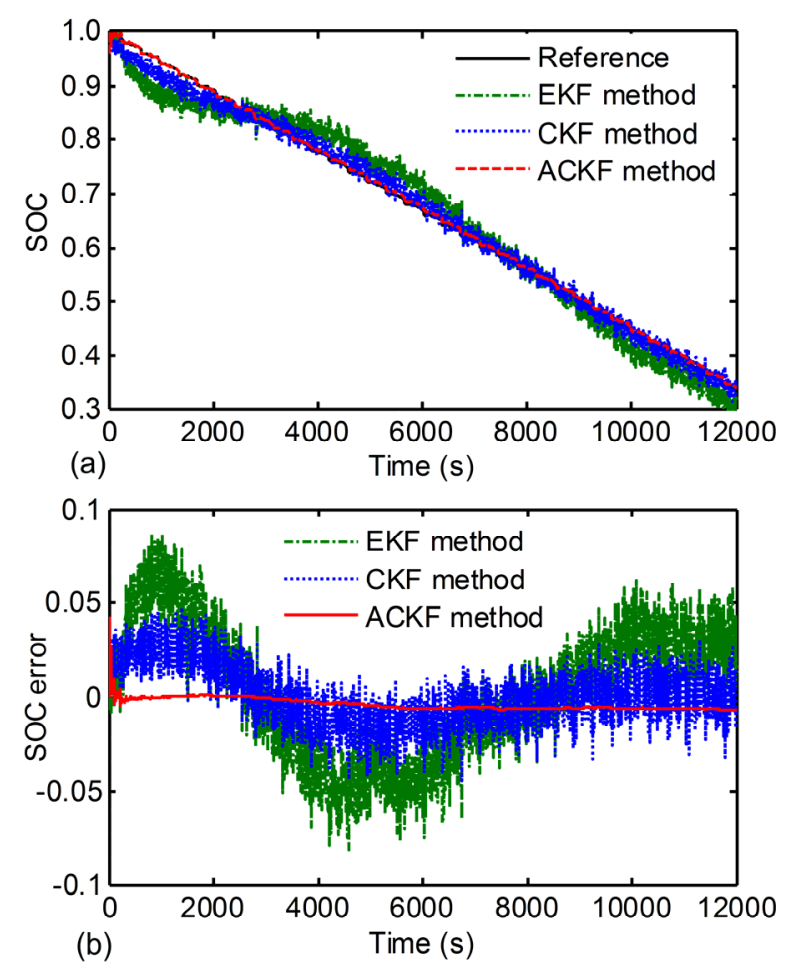

Figure 12. SOC estimation results with voltage noise under DST test: (a) SOC; (b) SOC error.
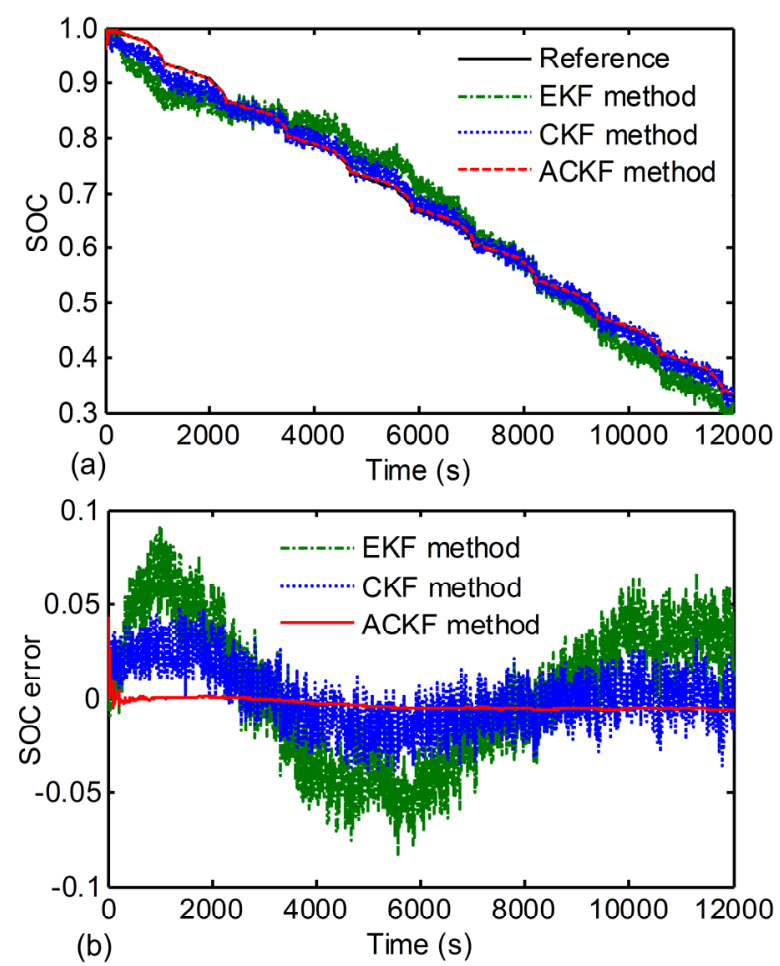

Figure 13. SOC estimation results with voltage noise under NEDC test: (a) SOC; (b) SOC error.

\section{Conclusions}

In this paper, an Adaptive Cubature Kalman filter (ACKF) algorithm is presented to accurately estimate SOC of the lithium-ion batteries in electric vehicles. The commonly used second-order RC equivalent circuit is applied to simulate the nonlinear behaviors of the lithium-ion battery and establish 
the battery state-space equations. The OCV-SOC relationship is fitted using the sixth-order polynomial and the other RC parameters of the battery model are determined by the forgetting factor least-squares algorithm. The principle of adaptive cubature Kalman filter for battery SOC estimation is introduced and the estimated process is presented in detail. Two typical driving cycles, including the Dynamic Stress Test and New European Driving Cycle, are applied to assess the performance of the proposed method by comparing with the traditional EKF and CKF algorithms. Experimental results indicate that, although the proposed ACKF algorithm takes more computational time compared with EKF and CKF, it is helpful to improve the SOC estimation accuracy and convergence to different initial SOC error. Furthermore, it is more robust against voltage measurement noise than EKF and CKF.

\section{Acknowledgments}

This work was simultaneously supported by the China Postdoctoral Science Foundation Funded Project (No. 2013M540941) and the Shenzhen Key Laboratory of LED Packaging Funded Project (No. NZDSY20120619141243215).

\section{Author Contributions}

Bizhong Xia and Haiqing Wang developed the essential idea behind the present research and prepared the manuscript in the early stages. Mingwang Wang, Wei Sun and Zhihui Xu established the test bench and carried out the experiments. Final review, including final manuscript corrections, was done by Yong Tian.

\section{Conflicts of Interest}

The authors declare no conflict of interest.

\section{References}

1. Aylor, J.H.; Thieme, A.; Johnson, B.W. A battery state-of-charge indicator for electric wheelchairs. IEEE Trans. Ind. Electron. 1992, 39, 398-409.

2. Ng, K.; Moo, C.S.; Chen, Y.P.; Hsieh, Y.C. Enhanced coulomb counting method for estimating state-of-charge and state-of-health of lithium-ion batteries. Appl. Energy 2009, 86, 1506-1511.

3. Lee, S.; Kim, J.; Lee, J.; Cho, B.H. State-of-charge and capacity estimation of lithium-ion battery using a new open-circuit voltage versus state-of-charge. J. Power Sources 2008, 185, 1367-1373.

4. Xing, Y.J.; He, W.; Pecht, M.; Tsui, K.L. State of charge estimation of lithium-ion batteries using the open-circuit voltage at various ambient temperatures. Appl. Energy 2014, 113, 106-115.

5. Feng, F.; Lu, R.G.; Wei, G.; Zhu, C.B. Online estimation of model parameters and state of charge of $\mathrm{LiFePO}_{4}$ batteries using a novel open-circuit voltage at various ambient temperatures. Energies 2015, 8, 2950-2976.

6. Do, D.V.; Forgez, C.; El Kadri Benkara, K.; Friedrich, G. Impedance observer for a Li-ion battery using Kalman filter. IEEE Trans. Veh. Technol 2009, 58, 3930-3937.

7. Xu, J.; Mi, C.C.; Cao, B.G.; Cao, J.Y. A new method to estimate the state of charge of lithium-ion batteries based on the battery impedance model. J. Power Sources 2013, 233, 277-284. 
8. Shen, Y.Q. Adaptive online state-of-charge determination based on neuro-controller and neural network. Energy Convers. Manag. 2010, 51, 1093-1098.

9. Cheng, B.; Bai, Z.F; Gao, B.G. State of charge estimation based on evolutionary neural network. Energy Convers. Manag. 2008, 49, 2788-2794.

10. Charkhgard, M.; Farrokhi, M. State-of-charge estimation for lithium-ion batteries using neural networks and EKF. IEEE Trans. Ind. Electron. 2010, 57, 4178-4187.

11. Dai, H.F.; Guo, P.J.; Wei, X.Z.; Sun, Z.C.; Wang, J.Y. ANFIS (adaptive neuro-fuzzy inference system) based online SOC (State of Charge) correction considering cell divergence for the EV (electric vehicle) traction batteries. Energy 2015, 80, 350-360.

12. Salkind, A.J.; Fennie, C.; Singh, P.; Atwater, T.; Reisne, D.E. Determination of state-of-charge and state-of-health of batteries by fuzzy logic methodology. J. Power Sources 1999, 80, 293-300.

13. Singh, P.; Vinjamuri, R.R.; Wang, X.Q.; Reisner, D. Design and implementation of a fuzzy logic-based state-of-charge meter for li-ion batteries used in portable defibrillators. J. Power Sources 2006, 162, 829-836.

14. Cuma, M.U.; Koroglu, T. A comprehensive review on estimation strategies used in hybrid and battery electric vehicles. Renew. Sustain. Energy Rev. 2015, 42, 517-531.

15. Plett, G.L. Extended Kalman filtering for battery management systems of LiPB-based HEV battery packs: Part 1. Background. J. Power Sources 2004, 134, 252-261.

16. Plett, G.L. Extended Kalman filtering for battery management systems of LiPB-based HEV battery packs: Part 2. Modeling and identification. J. Power Sources 2004, 134, 262-276.

17. Plett, G.L. Extended Kalman filtering for battery management systems of LiPB-based HEV battery packs: Part 3. State and parameter estimation. J. Power Sources 2004, 134, 277-292.

18. Lee, J.; Nam, O.; Cho, B.H. Li-ion battery SOC estimation method based on the reduced order extended Kalman filtering. J. Power Sources 2007, 174, 9-15.

19. Yuan, S.F.; Wu, H.J.; Yin, C.L. State of charge estimation using the extended Kalman filter for battery management systems based on the ARX battery model. Energies 2013, 6, 444-470.

20. Dai, H.F.; Wei, X.Z.; Sun, Z.C.; Wang, J.Y.; Gu, W.J. Online cell SOC estimation of Li-ion battery packs using a dual time-scale Kalman filtering for EV applications. Appl. Energy 2012, 95, 227-237.

21. Hu, C.; Youn, B.D.; Chung, J. A multiscale framework with extended Kalman filter for lithium-ion battery SOC and capacity estimation. Appl. Energy 2012, 92, 694-704.

22. Xiong, B.Y.; Zhao, J.Y.; Wei, Z.B.; Skyllas-Kazacos, M. Extended kalman filter method for state of charge estimation of vanadium redox flow battery using thermal-dependent electrical model. J. Power Sources 2014, 262, 50-61.

23. Sepasi, S.; Roose, L.R.; Matsuura, M.M. Extended kalman filter with a fuzzy method for accurate battery pack state of charge estimation. Energies 2015, 8, 5217-5233.

24. Xiong, R.; Gong, X.Z.; Mi, C.C.; Sun, F.C. A robust state-of-charge estimator for multiple types of lithium-ion batteries using adaptive extended Kalman filter. J. Power Sources 2013, 243, 805-816.

25. Sepasi, S.; Ghorbani, R.; Liaw, B.Y. A novel on-board state-of-charge estimation method for aged Li-ion batteries based on model adaptive extended Kalman filter. J. Power Sources 2014, 245, $337-344$. 
26. He, H.W.; Xiong, R.; Zhang, X.W.; Sun, F.C.; Fan, J.X. State-of-charge estimation of the lithium-ion battery using an adaptive extended Kalman filter based on an improved Thevenin model. IEEE Trans. Veh. Technol. 2011, 60, 1461-1469.

27. Zhang, C.; Li, K.; Pei, L.; Zhu, C.B. An integrated approach for real-time model-based state-of-charge estimation of lithium-ion batteries. J. Power Sources 2015, 283, 24-36.

28. Lee, S.J.; Kim, J.H. Discrete wavelet transform-based denoising technique for advanced state-of-charge estimator of a lithium-ion battery in electric vehicles. Energy 2015, 83, 462-473.

29. Plett, G.L. Sigma-point Kalman filtering for battery management systems of LiPB-based HEV battery packs: Part 1: Introduction and state estimation. J. Power Sources 2006, 161, 1356-1368.

30. Plett, G.L. Sigma-point Kalman filtering for battery management systems of LiPB-based HEV battery packs: Part 2: simultaneous state and parameter estimation. J. Power Sources 2006, 161, 1369-1384.

31. Sun, F.C.; Hu, X.S.; Zou, Y.; Li, S.G. Adaptive unscented Kalman filtering for state of charge estimation of a lithium-ion battery for electric vehicles. Energy 2011, 36, 3531-3540.

32. He, Z.W.; Gao, M.Y.; Wang, C.S.; Wang, L.Y.; Liu, Y.Y. Adaptive state of charge estimation for Li-ion batteries based on an unscented Kalman filter with an enhanced battery model. Energies 2013, 6, 4134-4151.

33. He, W.; Williard, N.; Chen, C.C.; Pecht, M. State of charge estimation for electric vehicle batteries using unscented Kalman filtering. Microelectron. Reliab. 2013, 53, 840-847.

34. Tian, Y.; Xia, B.Z.; Sun, W.; Xu, Z.H.; Zheng, W.W. A modified model based state of charge estimation of power lithium-ion batteries using unscented Kalman filter. J. Power Sources 2014, 270, 619-626.

35. Partovibakhsh, M.; Liu, G.J. An adaptive unscented Kalman filtering approach for online estimation of model parameters and state-of-charge of Lithium-ion batteries for autonomous mobile robots. IEEE Trans. Control. Syst. Technol. 2015, 23, 357-363.

36. Li, D.; Ouyang, J.; Li, H.Q.; Wan, J.F. State of charge estimation for $\mathrm{LiMn}_{2} \mathrm{O}_{4}$ power based on strong tracking sigma point Kalman filter. J. Power Sources 2015, 279, 439-449.

37. He, H.W.; Qin, H.Z.; Sun, X.K.; Shui, Y.P. Comparison study on the battery SoC estimation with EKF and UKF algorithms. Energies 2013, 6, 5088-5100.

38. Li, J.H.; Barillas, J.K.; Guenther, C.; Danzer, M.A. A comparative study of state of charge estimation algorithms for LiFePO4 batteries used in electric vehicles. J. Power Sources 2013, 230, 244-250.

39. Arasaratnam, I.; Haykin, S. Cubature Kalman filters. IEEE Trans. Autom. Control. 2009, 54, 1254-1269.

40. Arasaratnam, I.; Haykin, S.; Hurd, T.R. Cubature Kalman filtering for continuous-discrete systems: theory and simulations. IEEE Trans. Signal Process. 2010, 58, 4977-4993.

41. Arasaratnam, I.; Haykin, S. Cubature Kalman smoothers. Automatica 2011, 47, 2245-2250.

42. Li, W.; Jia, Y. Location of mobile station with maneuvers using an IMM-based cubature Kalman filter. IEEE Trans. Ind. Electron. 2012, 59, 4338-4348.

43. Dahmahi, M.; Meche, A.; Keche, M.; Oramri, A. Reduced cubature Kalman filtering applied to target tracking. In Proceedings of the 2nd International Conference on Control, Instrumentation and Automation (ICCIA'11), Shiraz, Iran, 27-29 December 2011; pp. 1097-1101. 
44. Tang, X.J.; Liu, Z.B.; Zhang, J.S. Square-root quaternion cubature Kalman filtering for spacecraft attitude estimation. Acta Astronautica 2012, 76, 84-94.

45. Guo, R.; Shi, P.F.; Cheng, X.Q.; Ma, Y.L.; Tan, Z. Effect of Ag additive on the performance of $\mathrm{LiNi}_{1 / 3} \mathrm{Co}_{1 / 3} \mathrm{Mn}_{1 / 3} \mathrm{O}_{2}$ cathode material for lithium ion battery. $J$ Power Sources 2009, 189, $2-8$.

46. Mousavi, G.S.M.; Nikdel, M. Various battery models for various simulation studies and applications. Renew. Sustain. Energy Rev. 2014, 32, 477-485.

47. Tian, Y.; Xia, B.Z.; Wang, M.W.; Sun, W.; Xu, Z.H. Comparison study on two model-based adaptive algorithms for SOC estimation of lithium-ion batteries in electric vehicles. Energies 2014, 7, 8446-8464.

48. Hu, X.S.; Li, S.B.; Peng, H. A comparative study of equivalent circuit models for Li-ion batteries. J. Power Sources 2012, 198, 359-367.

49. Tian, Y.; Chen, C.R.; Xia, B.Z.; Sun, W.; Xu, Z.H.; Zheng, W.W. An adaptive gain nonlinear observer for state of charge estimation of lithium-ion batteries in electric vehicles. Energies 2014, 7, 5995-6012.

50. Ogata, K. Discrete-Time Control Systems, 2nd ed.; Prentice Hall: Upper Saddle River, NJ, USA, 1995.

51. Feng, D.; Chen, T.W. Performance bounds of forgetting factor least-squares algorithms for time-varying systems with finite measurement data. IEEE Trans. Circuits Syst. Regul. Pap. 2005, $52,555-566$.

52. Pathuri Bhuvana, V.; Unterrieder, C.; Huemer, M. Battery internal state estimation: A comparative study of non-linear state estimation algorithms. In Proceedings of the 9th IEEE Vehicle Power and Propulsion Conference (IEEE VPPC'13), Beijing, China, 15-18 October 2013; pp. 65-70.

53. Mohamed, A.H.; Schwarz, K.P. Adaptive Kalman filtering for INS/GPS. J. Geod. 1999, 73, 193-203.

(C) 2015 by the authors; licensee MDPI, Basel, Switzerland. This article is an open access article distributed under the terms and conditions of the Creative Commons Attribution license (http://creativecommons.org/licenses/by/4.0/). 